ORIGINAL ARTICLE

\title{
Evaluation of Endometrial Scratching By Hysteroscope or Pipelle Curette on The Outcome of Assisted Reproduction
}

\author{
Eman Mahfouz Hafez, Entesar Roshdy Mahdy, Mohamed Abdel-Moniem Ibrahem, Manar \\ Mohamed Tharwat Emam Ahmed \\ Obstetrics \& Gynecology Department
}

Corresponding author :

Manar Mohamed Tharwat

Emam Ahmed

Email:

dr.manartharwat@gmail.com

$\begin{array}{ll}\text { Submit Date } & 2019-05-16 \\ \text { Revise Date } & 2019-06-20 \\ \text { Accept Date } & 2019-06-22\end{array}$

\begin{abstract}
Introduction:Endometrial scratching (or injury) is defined as intentional damage to the endometrium. The objective of this study was to examine the effect of the technique of endometrial scratching by hysteroscope or pipelle curette on the outcome of assisted reproduction. Scratching had done in the follicular phase of the previous cycle to embryo transfer in infertile ladies aged 20-40 years.
\end{abstract}

Methods:In this study, 225 ICSI cycles were considered and distributed into three groups: - hysteroscope group (75) scratching by scissor, pipelle group (75) scratching by pipelle curette, control group (75) no scratching had done. For each female in hysteroscope and pipelle groups an appointment was arranged on day7-14 of the cycle before the planned start of COS to do endometrial scratching.

Results:There were statistical significant differences between females in the pipelle group, hysteroscope group and control group as regard positive pregnancy test $\mathrm{P}$ value $=0.046$, clinical pregnancy $\mathrm{P}$ value $=$ 0.042 , ongoing pregnancy $\mathrm{P}$ value $=0.039$ and implantation rate $\mathrm{P}$ value $=0.044$. With further analysis to each intervention group independently with control group, there were statistical significant differences between females in the pipelle group and control group. There was improvement in pregnancy rates in hysteroscope group than control group but with no statistical significant differences.

Conclusion:Endometrial scratching achieved in the follicular phase of the earlier cycle to ovarian stimulation by pipelle curette or hysteroscope is linked with improved clinical and ongoing pregnancy rates in infertile females undergoing ICSI

Key words: Endometrial scratching, ICSI,Endometrium,Endometrial receptivity

\section{INTRODUCTION}

A ssisted reproductive techniques (ART) are used commonly to treat fertility complications, which affect roughly $7-15 \%$ of females of reproductive age. Although there have been numerous advances in techniques during the last three decades, clinical pregnancy and live-birth rates stay at approximately $30-40 \%$ and $20-30 \%$, correspondingly(1). Increasing these rates is desired for couples undergoing ART, as treatment failure is a source of psychological suffering and the most common cause of drop-out before attaining pregnancy. The most common interferences to improve ART outcome are the use of maximized controlled ovarian stimulation (COS), transfer of multiple embryos into the uterus and cryopreservation of excess oocytes/embryos. However, the first two of 
these interferences might increase the danger of ovarian hyperstimulation syndrome and multiple pregnancies, and there are ethical and economic concerns regarding oocyte/embryo cryopreservation (2).

Endometrial scratching (or injury) is defined as intentional damage to the endometrium. A connection between endometrial scratching and increased chance of pregnancy in subsequent ART procedures was defined a decade ago (3).

The endometrium is a complex tissue with divergent cellular compartments, including epithelial, stromal, endothelial cells and leukocytes, and also a dynamic tissue that goes through a sequence of morphological, biochemical, and molecular changes throughout the menstrual cycle (4).

Implantation is a multistage process including apposition and adhesion of the blastocyst to the uterine endometrium followed by invasion of the trophoblast among its epithelial cells (3).

Though the underlying mechanism remains unknown, hypotheses include:

- Endometrial scratching during the previous cycle might induce decidualization, increasing the chance of implantation.

- It induces a significant rise in the secretion of cytokines, interleukins, growth factors, macrophages and dendritic cells, all of which might be beneficial to embryo implantation.

- Endometrial scratching might lead to better synchronicity between the endometrium and the transferred embryo (2).

The aim of this work is to assess the influence of endometrial scratching by hysteroscope using scissors or pipelle curette in the non-transfer cycle to improve the chance of pregnancy in the subsequent first ICSI cycle.

PATIENTS AND METHODS Type of the study:
Cohort study (retro-prospective and record based data). The study was carried out in Obstetrics and Gynecology Department of Zagazig university hospitals and a private infertility center during the period from June 2016 to July 2018. Control group obtained from record based data (retrospective from June 2016 to December 2016) while intervention groups were randomized controlled trials (prospective from January 2017 to July 2018).

The study was conducted on infertile females aged 20- 40 years with normal uterine cavity undergoing the first ICSI cycle. Excluded cases were females who had any uterine anomaly or pathology, recurrent implantation failure, visible hydrosalpinx on transvaginal ultrasound scans, azoospermia and medical disorders as hypertention, diabetes, endocrine diseases, liver and renal diseases.

All patients were subjected to Full history taking, general, abdominal and local examinations were done to exclude cases with any abnormality. Hepatitis marker (HBsAg, HCAb). Routine preoperative investigations $(\mathrm{CBC}$, coagulation profile, LFT, KFT, blood group and random blood sugar), abdominal US, transvaginal US and hormonal profile: Follicle-stimulating hormone (FSH), Luteinizing hormon(LH), Thyroid-stimulating hormone (TSH) , Testosterone and Prolactin.

\section{Study design}

The required sample size has been calculated by Open Epi assuming that implantation rate in females submitted to endometrial scratching group is $35.5 \%$ and in control group is $18.8 \%$, confidence level $95 \%$ and power $80 \%$ so that total sample size was 225 case and was divided into three groups ; hysteroscope group (75), pipelle group (75) and control group (75).

For each woman in hysteroscope and pipelle groups an appointment was 
arranged in day 7-14 of the cycle before the planned start of COS.

\section{Group 1 The Hysteroscope group}

A rigid 30degree 4-mm hysteroscope (Karl Storz Endoscopy) was used with anesthesia when indicated (stenosis of internal os and uncooperative patient). The uterine cavity was distended with normal saline solution at a pressure of 100-120 mmHg. The vaginoscopic "no touch" technique was followed; no speculum or tenaculum was used. Endometrial scratching was done to the uterine fundus between two tubal ostia using a scissor as three cuttings of $0.5 \mathrm{~cm}$ on the front endometrial wall, 1 $\mathrm{cm}$ lower of the endometrial fundus level. No antibiotics were given after the procedure.

\section{Group 2 The Pipelle group}

On the planned day, the participants were ready for a gynecological examination and the uterine cervix was identified using a vaginal speculum, endometrial scratching was achieved once with a pipelle de Cornier (Laboratoires Prodimed, Neully-En-Thelle, France). The pipelle was introduced gently through the cervix up to the uterine fundus. The piston was then drawn back to the end of the biopsy cannula until it self-locked, creating a negative pressure. Directing to cover the entire endometrium, the examiner applied regular back-and-forth movements through anterior, posterior and lateral uterine walls.

Group 3 The Control group: no scratching was done

On COS start day, females underwent transvaginal ultrasound examination to exclude ovarian cyst; COS was started with long, short or antagonist protocol.

In long Protocol daily injection of a GnRH agonist (Decapeptyl® $0.1 \mathrm{mg}$; Ferring, Germany) administered by S.C. injection, is initiated in the mid-luteal phase of the preceding cycle (day 21). Patients present on day 3 of the following menstrual cycle for baseline serum E2 level and ultrasound (US) assessment. Upon determining adequate pituitary suppression, suitably suppressed E2 level < 50 pgm, a thin endometrial stripe $<5 \mathrm{~mm}$ by US and no cyst, daily HMG is started 150 - $300 \mathrm{IU}$ (Fostimon ${ }^{\circledR} 75 \mathrm{IU}$; IBSA, Farmaceutici, Italia, S.r). TVS observing of follicle development was scheduled to start after 5 days of COS, repeating every other day. In short "flare up" protocol administration of a GnRH agonist (Decapeptyl® $0.1 \quad \mathrm{mg}$ ;Ferring, Germany) in day 2 of cycle. High-dose gonadotropin (225-450 IU) (Fostimon ${ }^{\circledR} 75$ IU; IBSA, Farmaceutici, Italia, S.r) stimulation is usually begun on cycle day 3. TVS observing of follicle development was scheduled to start after 5 days of COS, repeating every other day. In GnRH Antagonist protocol daily HMG is started (150 -225 IU) from day 2 of the cycle (Fostimon ${ }^{\circledR} \quad 75$ IU;IBSA, Farmaceutici, Italia, S.r). TVS observing of follicle development was scheduled on day 8 repeating every other day. U/S showed one follicle $\geq 14 \mathrm{~mm}$, daily $\mathrm{GnRH}$ antagonist is administered subcutaneously (Cetrotide $0.25 \mathrm{mg}$; Merck KGaA, Frankfurter Straße 250, D-64293 Darmstadt, Germany).

Final follicular maturation in any protocol was triggered when two follicles $\geq 18 \mathrm{~mm}$, using human chorionic gonadotropin 10000 IU single injection (choriomon 5000 IU; IBSA, Farmaceutici, Italia, S.r).

Oocyte retrieval was achieved 34-36 h after the hCG injection. From the day of retrieval, the luteal phase was maintained by the administration of Progesterone (Crinone 8\% Vaginal gel; Fleet Laboratories LimitedUnited Kingdom: Serono Limited - United Kingdom) once daily or (cyclogest 400mg vaginal pessaries; Actavis, United Kingdom) twice daily.

One to three embryos were transferred on day 3 after oocyte retrieval. Two weeks after 
embryo transfer, serum human chorionic gonadotropin (hCG) was measured for approval of pregnancy \& diagnosis of clinical pregnancy was made after visualization of fetal heart pulsation four weeks later by TVS. Luteal phase support was stopped if the pregnancy test was negative or after week 12 of pregnancy.

\section{Outcomes}

The main outcome measures were the implantation, clinical pregnancy, and ongoing pregnancy rates. The secondary outcome measures were multiple pregnancy and miscarriage rates. Implantation rate was defined as the number of gestational sacs detected, divided by the number of embryos transferred. Clinical pregnancy was defined as the presence of a sac and fetal heart pulsation by transvaginal USG on the 6th gestational week. Ongoing pregnancy was defined as the presence of at least one fetal heart pulsation on or beyond the 12 weeks of gestation by US(5).

\section{Administrative design:}

Written informed consent was obtained from all participants and the study was approved by the research ethical committee of Faculty of Medicine, Zagazig University. The work has been carried out in accordance with The Code of Ethics of the World Medical Association (Declaration of Helsinki) for studies involving humans.

\section{Statistical Methods}

Data were analyzed using IBM@ SPSSC Statistics version 22 (IBMC Corp., Armonk, NY, USA) and MedCalc $($ version 13 (MedCalc@ Software bvba, Ostend, Belgium). Continuous numerical variables were presented as mean and SD, and intergroup differences were compared using one-way analysis of variance (ANOVA) (for multiple-group comparison).

Discrete numerical variables were presented as median and interquartile range and between-group differences were compared using the Kruskal-Wallis test (for multiple- group comparison). Categorical data were presented as number and percentage or as ratio and differences were compared using the Pearson chi-squared test. A two-sided pvalue $<0.05$ was considered statistically significant.

\section{RESULTS}

During the study period 270 case started for ICSI, 30 cases were excluded from the study not meeting inclusion criteria. 5 cases cancelled due to poor ovarian response, 10 cases had freeze all embryos and no transfer occurred due to fear of OHSS. So total sample size of the study was 225 case.

This cohort retro-prospective study evaluated the effect of endometrial scratching by two different methods; hysteroscope with scissor or pipelle curette in day (7-14) of the preceding cycle to ovarian stimulation on pregnancy rate in females with infertility undergoing ICSI. The study compared between pipelle group , hystroscope group and control group. Participants' characteristics (Tab.1) showed no statistical significant differences between females in the pipelle group, hysteroscope group and control group as regard mean age, BMI, type of infertility, basal FSH, LH and E2 ( $\mathrm{P}$ value >0.05). In ICSI cycles, also there were no statistical significant differences between females in protocol used, number of retrieved oocytes, number of injected oocytes, number of transferred embryos and number of grade A embryos transferred ( $\mathrm{P}$ value $>0.05$ ) (Tab.2).

In this study, Implantation rate was found to be higher in pipelle group 50/157(31.8\%) and in hysteroscope group was $36 / 156(23.1 \%)$ compared to $27 / 158(17.1 \%)$ in control group. The difference was statistically significant $\mathrm{P}$ value $<0.05=$ 0.044 .

As well as, cases with positive pregnancy test in pipelle group were (39/75) and in hysteroscope group were (32/75) compared with the control group (24/75) [52\%, 
$42.7 \%$ vs.32\% respectively] and the difference was statistically significant $\mathrm{p}$ value $<0.05=0.046$.

As regard chemical pregnancy in the present study, it was (2/39) in pipelle group and in hysteroscope group (1/32) compared with control group $(2 / 24)[5.1 \%, 3.1 \%$ vs. $8.3 \%$ respectively] $\mathrm{p}$ value $>0.05=0.688$ so there was no significant difference between groups.

Also, clinical pregnancy rate in this study was significantly higher in females in pipelle group (37/75) and in hysteroscope group (31/75) compared with control group $(22 / 75) \quad[49.3 \%, \quad 41.3 \%$ vs. $29.3 \%$ respectively] $\mathrm{p}$ value $<0.05=0.042$. There was no evidence of effect on abortion in this study as there was no statistical significant difference in abortion rate in the study groups $\mathrm{P}=0.859$. Also there was no statistical significant difference in multiple pregnancy in the study groups $\mathrm{P}$ value $>0.05$ $=0.731$.

Furthermore ongoing pregnancy rate in this study was significantly higher in females subjected to endometrial scratching by pipelle (35/75) and by hysteroscope (29/75) compared with control group (20/75) [46.7\%, $38.7 \%$ vs. $26.6 \%$ respectively] p value $<0.05=0.039$.

While comparing the outcome measures of the two scratching groups, the results of pipelle group were higher than hysteroscope group but not statistically significant (Tab.4). Positive pregnancy test in pipelle group to hysteroscope group was $39(52 \%)$, $32(42.7 \%)$ respectively .Clinical pregnancy rate was $37(49.3 \%), 31(41.3 \%)$ respectively. Ongoing pregnancy rate was $35(48 \%)$, $29(38.7 \%)$ respectively.

Table (1): participants' characteristics in the pipelle, hystroscope and control groups.

\begin{tabular}{|l|l|l|l|l|}
\hline Variable & $\begin{array}{l}\text { Pipelle } \\
\text { Group } \\
(\mathbf{n = 7 5})\end{array}$ & $\begin{array}{l}\text { Hystroscope } \\
\text { Group }(\mathbf{n = 7 5})\end{array}$ & $\begin{array}{l}\text { Control } \\
\text { Group } \\
(\mathbf{n = 7 5})\end{array}$ & p-value \\
\hline Age $(\mathrm{yr})$ & $9.36 \pm(5.2)$ & $31.38 \pm(4.8)$ & $30.76 \pm(4.9)$ & $0.125 \llbracket$ \\
\hline BMI $(\mathrm{kg} / \mathrm{m} 2)$ & $8.2 \pm(3.9)$ & $28.2 \pm 3.1$ & $28.2 \pm 3.1$ & $0.983 \llbracket$ \\
\hline Type of Infertility 1ry/2ry & $51 / 24$ & $55 / 20$ & $45 / 30$ & $0.216 \S$ \\
\hline Basal FSH (mIU/ml) & $6.9 \pm 1.4$ & $7.0 \pm 1.9$ & $6.8 \pm 1.5$ & $0.715 \llbracket$ \\
\hline Basal LH $(\mathrm{mIU} / \mathrm{ml})$ & $5.4 \pm 1.3$ & $5.1 \pm 1.1$ & $5.5 \pm 1.0$ & $0.083 \llbracket$ \\
\hline Basal E2 $(\mathrm{pg} / \mathrm{ml})$ & $62.2 \pm 6.3$ & $62.1 \pm 6.3$ & $60.7 \pm 7.6$ & $0.313 \llbracket$ \\
\hline
\end{tabular}

Data are presented as mean (SD), ratio.

IIOne-way analysis of variance (ANOVA).

$\S$ Pearson chi-squared test. 
Table (2): Cycle stimulation characteristics in the pipelle, hystroscope and control groups.

\begin{tabular}{|c|c|c|c|c|}
\hline Variable & $\begin{array}{l}\text { Pipelle Group } \\
(n=75)\end{array}$ & $\begin{array}{l}\text { Hystroscope Group } \\
(n=75)\end{array}$ & $\begin{array}{l}\text { Control Group } \\
(n=75)\end{array}$ & p-value \\
\hline $\begin{array}{l}\text { Protocol } \\
\text { Long } \\
\text { Short } \\
\text { Antagonist }\end{array}$ & $\begin{array}{l}28(37.3 \%) \\
22(29.3 \%) \\
25(33.3 \%)\end{array}$ & $\begin{array}{l}24(32 \%) \\
21(28 \%) \\
30(40 \%)\end{array}$ & $\begin{array}{l}25(33.3 \%) \\
20(26.7 \%) \\
30(40 \%)\end{array}$ & $0.907 \S$ \\
\hline Number of retrieved oocyte & $12(4-20$ & $12(3-21)$ & $15(4-22)$ & $0.230 \mathbb{I}$ \\
\hline Number of injected oocytes & $9(3-19)$ & $9(2-20)$ & $10(2-19)$ & 0.309प \\
\hline $\begin{array}{l}\text { Number of transferred } \\
\text { embryos } \\
1 \\
2 \\
3\end{array}$ & $\begin{array}{l}\quad 2(1-3) \\
5 / 75(6.7 \%) \\
58 / 75(77.3 \%) \\
12 / 75(16 \%)\end{array}$ & $\begin{array}{l}2(1-3) \\
6 / 75(8 \%) \\
57 / 75(76 \%) \\
12 / 75(16 \%)\end{array}$ & $\begin{array}{l}2(1-3) \\
5 / 75(6.7 \%) \\
57 / 75(76 \%) \\
13 / 75(17.3 \%)\end{array}$ & $0.931 \Phi$ \\
\hline $\begin{array}{l}\text { Number of grade A embryo } \\
s \text { transferred }\end{array}$ & $2(1-3)$ & $2(1-3)$ & $2(1-3)$ & $0.127 \Phi$ \\
\hline
\end{tabular}

Data are presented as median (interquartile range), number (\%).

II Kruskal-Wallis test

$\S$ Pearson chi-squared test

Table (3): Comparison of the outcome measures in study groups

\begin{tabular}{|c|c|c|c|c|}
\hline Variable & $\begin{array}{l}\text { Pipelle group } \\
\qquad(\mathrm{n}=75)\end{array}$ & $\begin{array}{l}\text { Hysteroscope } \\
\text { group } \\
(n=75)\end{array}$ & $\begin{array}{c}\text { Control } \\
\text { group }(n=75)\end{array}$ & p-value \\
\hline Positive pregnancy test & $39(52 \%)$ & $32(42.7 \%)$ & $24(32 \%)$ & 0.046 II \\
\hline Chemical pregnancy & $2 / 39(5.1 \%)$ & $1 / 32(3.1 \%)$ & $2 / 24(8.3 \%)$ & 0.688 II \\
\hline Clinical pregnancy & $37(49.33 \%)$ & $31(41.33 \%)$ & $22(29.33 \%)$ & $0.042 \rrbracket$ \\
\hline Abortion & $2 / 37(5.4 \%)$ & $2 / 31(6.5 \%)$ & $2 / 22(9.1 \%)$ & $0.859 \mathrm{I}$ \\
\hline Ongoing pregnancy & $35(46.7 \%)$ & $29(38.7 \%)$ & $20(26.6 \%)$ & 0.039प \\
\hline Implantation rate & $50 / 157(31.8 \%)$ & $36 / 156(23.1 \%)$ & $\begin{array}{l}27 / 158 \\
(17.1 \%)\end{array}$ & $0.044 \rrbracket$ \\
\hline
\end{tabular}

Data are presented as number $(\%)$.

II Pearson chi-squared test 
Table (4): Comparison of the outcome measures in scratching groups

\begin{tabular}{|l|l|l|l|}
\hline Variable & $\begin{array}{l}\text { Pipelle group } \\
(\mathbf{n = 7 5})\end{array}$ & $\begin{array}{l}\text { Hysteroscope } \\
\text { group }(\mathbf{n}=\mathbf{7 5})\end{array}$ & P value \\
\hline Positive pregnancy test & $39(52 \%)$ & $32(42.7 \%)$ & $0.252 \mathrm{I}$ \\
\hline Chemical pregnancy & $2 / 39(5.1 \%)$ & $1 / 32(3.1 \%)$ & $0.676 \mathrm{I}$ \\
\hline Clinical pregnancy & $37(49.33 \%)$ & $31(41.33 \%)$ & $0.325 \mathrm{I}$ \\
\hline Abortion & $2 / 37(5.4 \%)$ & $2 / 31(6.5 \%)$ & $0.855 \mathrm{I}$ \\
\hline Ongoing pregnancy & $35(46.7 \%)$ & $29(38.7 \%)$ & $0.322 \mathrm{I}$ \\
\hline
\end{tabular}

Data are presented as number (\%).

II Pearson chi-squared test

Table (5): Comparison of the outcome measures in Pipelle and control groups

\begin{tabular}{|l|l|l|l|}
\hline Variable & $\begin{array}{l}\text { Pipelle group } \\
(\mathbf{n = 7 5})\end{array}$ & $\begin{array}{l}\text { Control group } \\
(\mathbf{n = 7 5})\end{array}$ & P value \\
\hline Positive pregnancy test & $39(52 \%)$ & $24(32 \%)$ & $0.013 \mathrm{II}$ \\
\hline Chemical pregnancy & $2 / 39(5.1 \%)$ & $2 / 24(8.3 \%)$ & 0.612 I \\
\hline Clinical pregnancy & $37(49.33 \%)$ & $22(29.33 \%)$ & 0.012 I \\
\hline Abortion & $2 / 37(5.4 \%)$ & $2 / 22(9.1 \%)$ & 0.586 I \\
\hline Ongoing pregnancy & $35(46.7 \%)$ & $20(26.7 \%)$ & 0.011 I
\end{tabular}

Data are presented as number (\%).

II Pearson chi-squared test

Table (6): Comparison of the outcome measures in Hysteroscope and control groups

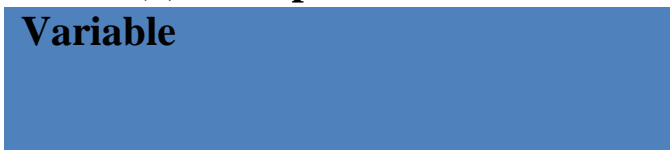

\begin{tabular}{l}
$\begin{array}{l}\text { Hysteroscope } \\
\text { group } \\
(\mathbf{n}=75)\end{array}$ \\
\hline $32(42.7 \%)$
\end{tabular}

$\begin{aligned} & \text { control group } \\ & (\mathbf{n}=75)\end{aligned}$
nat

P value

Positive pregnancy test

$32(42.7 \%)$

$24(32 \%)$

$2 / 24(8.3 \%)$

0.1779

Chemical pregnancy

$1 / 32(3.1 \%)$

$22(29.33 \%)$

$0.392 \mathbb{I}$

Clinical pregnancy

Abortion

Ongoing pregnancy

\begin{tabular}{|l|l|l|}
\hline $31(41.33 \%)$ & $22(29.33 \%)$ & $0.124 \mathbb{I}$ \\
\hline $2 / 31(6.5 \%)$ & $2 / 22(9.1 \%)$ & $0.720 \mathbb{I}$ \\
\hline $29(38.7 \%)$ & $20(26.7 \%)$ & $0.117 \mathrm{I}$
\end{tabular}

Data are presented as number (\%).

II Pearson chi-squared test 


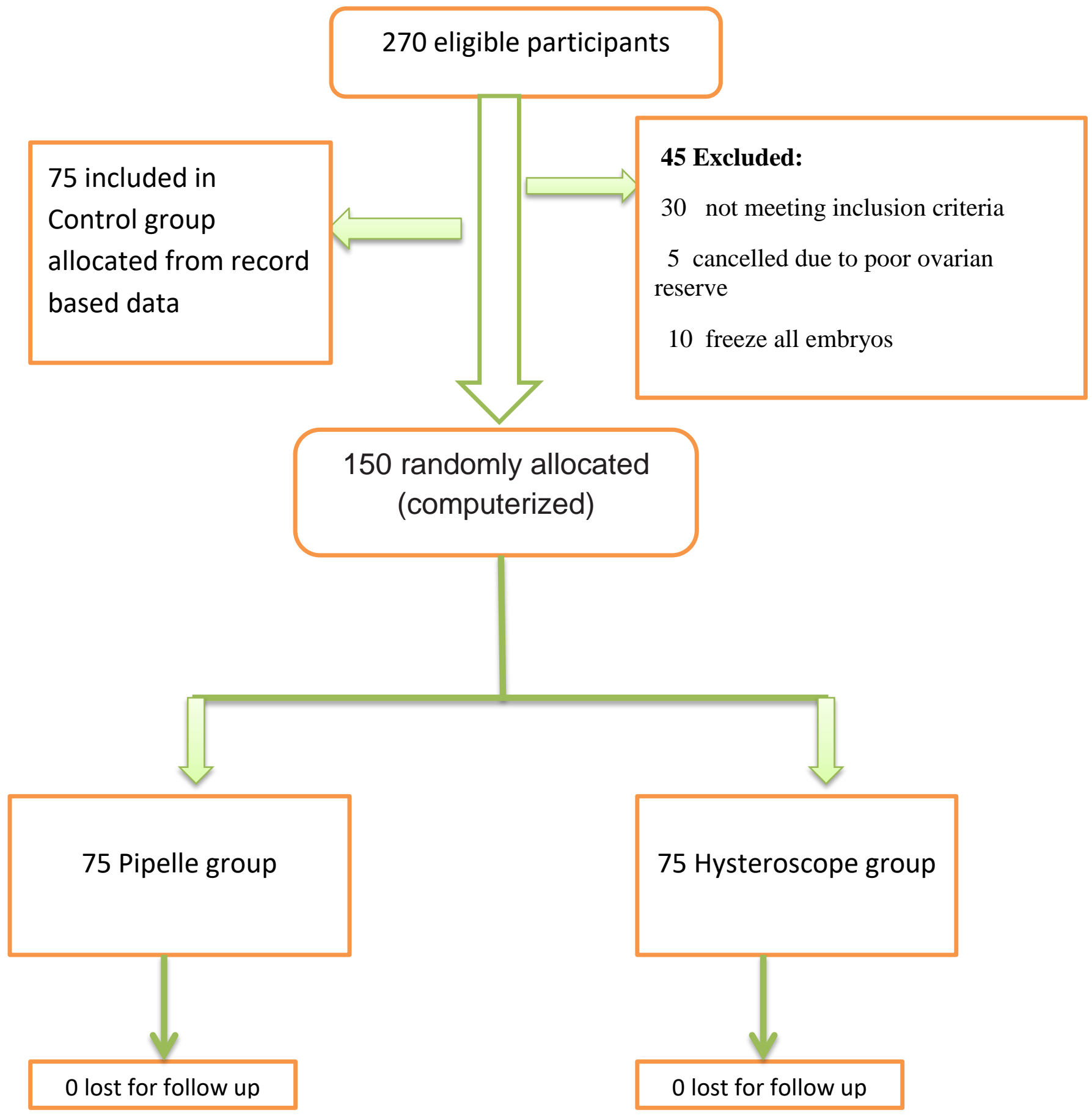

Figure 1: Participant flow 


\section{DISCUSSION}

The present study compared between two different methods of endometrial scratching and evaluated their impact on pregnancy outcome in infertile females undergoing ICSI. So the study compared pipelle group, hysteroscope group with control group separately (Tab.5,6).

This study results revealed that scratching by pipelle had improved pregnancy outcomes and there was a statistically significant difference with control group. Positive pregnancy test in pipelle group to control group was 39(52\%), 24(32\%) respectively .Clinical pregnancy rate was $37(49.3 \%), \quad 22(29.3 \%) \quad$ respectively. Ongoing pregnancy rate was 35(48\%), $20(26.7 \%)$ respectively p value $<0.05=0.01$. The results of scratching by hysteroscope using scissors were higher than those of control group but there was no statistically significant difference as $p$ value $>0.05=0.1$. Positive pregnancy test in hysteroscope group to control group was 32(42.7\%), $24(32 \%)$ respectively. Clinical pregnancy rate was 31(41.3\%), 22(29.3\%) respectively. Ongoing pregnancy rate was $29(38.7 \%)$, $20(26.7 \%)$ respectively. However there was improvement in pregnancy outcomes, there was no statistically significant difference as $\mathrm{p}$ value $>0.05=0$. 1 possibly due to small sample size group.

These results were agreed with Karimzadeh et al.,2009 in which a RCT conducted on 115 females with RIF divided into two groups randomly: an experimental group ( $\mathrm{n}$ $=58)$ and a control group $(\mathrm{n}=$ 57).Endometrial scratching was done in the luteal phase of the preceding cycle to ovarian stimulation by pipelle catheter . Implantation rate was found to be $10.9 \%$ in the intervention group compared to $3.38 \%$ in the controls, and the difference was statistically significant $(\mathrm{P}=0.039)(6)$. Also in Seval et al.,2016 study that was conducted on 345 females with RIF.
Females were allocated into two groups; scratching group by using monopolar electric energy with needle forceps during hysteroscopy, and control group in which a diagnostic hysteroscopy only was done with no scratching. The implantation rate was significantly higher in scratching group than control group $(37.7 \%$ versus $24.5 \%$; $\mathrm{p}=$ 0.04). Also clinical pregnancy rate in patients underwent endometrial scratching (65/171) was higher than rate in control group (48/171)[38\%vs27.6\% $\mathrm{P}=0.03](7)$.

Also Narvekar et al.,2010 had performed scratching 100 patients. The implantation rate was significantly higher in the intervention group as compared to control $(13.07 \%$ vs. $7.1 \% \quad \mathrm{P}=0.04)$. Clinical pregnancy rate in the intervention group was $32.7 \%$, while that in the control group was $13.7 \%,(\mathrm{P}=0.01)$. The live birth rate was significantly higher in the intervention group compared to control group (22.4\% and $9.8 \%$ $\mathrm{P}=0.04)(8)$.

Similarly the study by Guven et al, had performed endometrial scratching once and found a higher clinical pregnancy $(48.2 \%$ vs. $29.0 \%, \mathrm{P}=0.025)$ and live birth (33.9\% vs. $17.7 \%, \mathrm{P}=0.035)$ rates in the intervention group compared with control group(9).

Endometrial scratching in the follicular phase of the previous cycle may allow adequate time for endometrial regeneration and proliferation to achieve the desired effect. In support of this theory Li et al, used an animal model to prove histologically that the endometrium requires approximately 2 weeks to attain complete repair after mechanical injury(10).

\section{CONCLUSION}

This study results detected that endometrial scratching achieved in the follicular phase of the previous cycle to ovarian stimulation by pipelle curette or hysteroscope is associated with improved clinical and ongoing pregnancy rates in infertile females 
undergoing ICSI through enhancing the endometrial receptivity. The significant difference was in pipelle group only while hysteroscope group was not significant compared with control. There was no statistical significant difference between the two methods.

\section{Declaration of interest}

The authors report no conflicts of interest. The authors alone are responsible for the content and writing of the paper.

Funding information None declared

\section{REFERENCES}

1. Audibert C, Glass D. A global perspective on assisted reproductive technology fertility treatment: an 8-country fertility specialist survey. Reproductive Biology and Endocrinology : RB\&E. 2015;13:133.

2. Nastri C, Ferriani R, Raine-Fenning N, Martins W. Endometrial scratching performed in the non-transfer cycle and outcome of assisted reproduction: a randomized controlled trial. Ultrasound in Obstetrics \& Gynecology. 2013;42(4):37582.

3. Barash A, Dekel N, Fieldust S, Segal I, Schechtman E, Granot I. Local injury to the endometrium doubles the incidence of successful pregnancies in patients undergoing in vitro fertilization. Fertility and sterility. 2003;79(6):1317-22.

4. Zhou L, Li R, Wang R, Huang H-x, Zhong $\mathrm{K}$. Local injury to the endometrium in controlled ovarian hyperstimulation cycles improves implantation rates. Fertility and sterility. 2008;89(5):1166-76.

5. Zegers-Hochschild F, Adamson GD, de Mouzon J, Ishihara O, Mansour R, Nygren $\mathrm{K}$, et al. The international committee for monitoring assisted reproductive technology (ICMART) and the world health organization
(WHO) revised glossary on ART terminology, 2009. Human Reproduction. 2009:dep343.

6. Karimzadeh MA, AYAZI ROZBAHANI M, Tabibnejad N. Endometrial local injury improves the pregnancy rate among recurrent implantation failure patients undergoing in vitro fertilisation/intra cytoplasmic sperm injection: a randomised clinical trial. Australian and New Zealand Journal of Obstetrics and Gynaecology. 2009;49(6):677-80.

7. Seval MM, Şükür YE, Özmen B, Kan Ö, Sönmezer M, Berker B, et al. Does adding endometrial scratching to diagnostic hysteroscopy improve pregnancy rates in females with recurrent in-vitro fertilization failure? Gynecological Endocrinology. 2016;32(12):957-60.

8. Narvekar SA, Gupta N, Shetty N, Kottur A, Srinivas M, Rao KA. Does local endometrial injury in the nontransfer cycle improve the IVF-ET outcome in the subsequent cycle in patients with previous unsuccessful IVF? A randomized controlled pilot study. Journal of human reproductive sciences. 2010;3(1):15.

9. Guven S, Kart C, Unsal MA, Yildirim O, Odaci E, Yulug E. Endometrial injury may increase the clinical pregnancy rate in normoresponders undergoing long agonist protocol ICSI cycles with single embryo transfer. European Journal of Obstetrics and Gynecology and Reproductive Biology. 2014;173:58-62.

10.Li L, Shi J, Zhang Q, Yan J, Yan L, Shen F, et al. Effect of curettage and copper wire on rabbit endometrium: a novel rabbit model of endometrial mechanical injury. Chinese medical journal. 2011;124(11):1708-13.

To Cite This Article: Hafez EM, Mahdy ER, Ibrahem MA, Ahmed MM. Evaluation of Endometrial Scratching By Hysteroscope or Pipelle Curette on The Outcome of Assisted Reproduction. ZUMJ 2020; 26 (2) 297-306; Doi: 10.21608/zumj.2019.12345.1216. 